\title{
Inequality in Training: Bridging the Gap
}

\section{Rohit Rambani ${ }^{1}$}

Published online: 31 July 2020

(c) Indian Orthopaedics Association 2020
During the testing time of COVID-19, the surgical training has been hit hard and a re-think is required how we should be training our surgical trainees. Surgical training has traditionally been based on the apprentice model. The transition from the internship to residency is often considered the most difficult year for both teachers and learners. There is an expectation that new doctors will perform to an acceptable level in their new clinical role, integrate into a clinical team, and take full responsibility for their actions starting on day one of training. However, research has shown that trainees often feel anxious, underprepared and stressed when entering residency, this leads to what is called as the 'February or August phenomenon' or a decrease in health care efficiency and patient safety during trainees' first month of training [1].

The gap in the assessment of the trainees leads to the huge discrepancy on the level of training each institute or hospital imparts to the particular trainee. This discrepancy becomes more obvious when training is compared between a premier institute and a peripheral hospital. In our current system of training repeated assessment is lacking and a lot to be desired for. A regular structured feedback to the trainee can help both the trainers and the trainee to identify the lacunae in training and thus a focused approach can be made to improve the competency of the trainee. Simulation has been identified as a means to increase trainee exposure to and experience with clinical tasks, without increasing the burden of patient harm.

Various countries used the competency-based assessment or the procedure-based assessment as a tool for progression of training. Competency-Based Medical Education (CBME) represents the biggest change to medical education since Halstead proposed his apprenticeship model over 100 years ago. This new paradigm has been crafted over nearly four decades, and is built on the principle that trainees and

Rohit Rambani

rohitrambani@gmail.com

1 Department of Orthopaedics, United Lincolnshire Hospital NHS Trust, Boston, UK surgeons must full-fill core competencies, spanning from ethics to professionalism to technical aptitude, prior to independent practice. Traditionally the standard of training varies vastly on the hospital or institute the training is imparted and thus the standard of surgeon coming out of the training program also varies.

One of the ways for the trainees to retain information is through is through repeated assessment. The act of retrieving the correct information from long-term memory repeatedly activates the neurons associated with the correct response and makes it easier to retrieve in the future. However, often learners are unable to recall the information successfully. Thus, regardless of whether learners answer correctly, testing (and retesting) can often be more powerful than re-studying information.

To decrease the gap of inequality in training from various institutes a repeated structured assessment system needs to be developed to assure that a minimum standard is expected from the trainee when he/she is exiting the training program. One such method is the development of boot camps across various regions. Boot camps at onset of training will contain a multitude of novel tasks that trainees must acquire. In addition to opportunities for early assessment of trainees, the surgical boot camp has been recognized as a means of introducing trainees to the unique culture of postgraduate surgical training. Subsequently the boot camps should be developed for each semester of the trainee to assess progression and impart new knowledge. To effectively teach these tasks, medical educators must consider what training methods are best for novice skill acquisition. To facilitate permanent learning, boot camps must involve repetitive practice, observational learning, distributed practice, frequent formative assessments, self-regulated feedback, and focused instruction towards extrinsic factors. Developing a boot camp founded in these principles offers an opportunity for trainees to improve their medical knowledge, confidence, and procedural and technical skills in a safe environment. Gaining this valuable exposure prior to starting to care for patients may extenuate trainee feelings of unpreparedness as well as patient complication rates. 
Furthermore, acquiring these basic skills at the onset of residency privileges progression through subsequent stages of training since less time and attention need to be dedicated to those basic skills [2]. Thus, if boot camps are aligned with the theoretical foundations of novice skill acquisition, this type of training algorithm has the potential to revolutionize medical training. The boot camp can be based on few key principles:

- A classroom model.

- Peer facilitators.

- Graduated complexity of tasks.

- Basic surgical principle training.

- Case based learning.

- Formative feedback.

To limit the need for didactic lecturing, and to allow for a case based and facilitator driven approach, an intense preparation is required from both the facilitators and trainees. At the beginning of the camp, the course outline and reading/ video list are distributed. Each day's lecture, skills and anatomy sessions build on this provided material. This changes the focus from a didactic experience to one where residents engage in a learning environment and problem solve together using their new knowledge.

Having a trained faculty to run the course is the key to the success. It is prudent that the trainers and facilitators understand the need of the trainees and make sure that the facilitators and assessors are aware what generic or specific skills they are assessing [3]. Increasing the level of complexity in learning the cognitive skills is individualized to each trainee and the progression is assessed by facilitators/trainers to let the trainee progress to the next level. This helps in avoiding the phenomenon of one fix for all. Case based learning is used to understand concepts. Cases are structured similarly to oral exam questions a resident might expect to find in their exit exam tailored to an early training level.

A repeated structured assessment during the training program will help assess the progression of the trainee and fine tune the training program to accommodate the training needs of the trainee.

Typical reasons for poor performance include the variable quantity and quality of technical skills training in medical school, trainee technical aptitudes, invested effort, and test-related anxiety. Examination design, and the individual examiner characteristics and training also contribute to residents' exam scores [4].
Finally, evidence must continue to be gathered, especially in the assessment of surgical as well as non-technical skills, to satisfy the public and stakeholders in the accreditation and certification of doctors/surgeons [5]. The need to narrow the gap in training standards is a must before a surgeon is allowed to do private practice and should be irrespective of the institute of hospital he has been trained. A centralized assessment similar to UK/US Exit exams along with regular formative assessment can help identify trainees those will need further training. The use of assessment tools that adhere to validity frameworks is essential to ensuring the acceptability of decisions made around trainee progression along the skill acquisition continuum.

\section{Compliance with ethical standards}

Conflict of interest The author declares no conflict of interest.

Ethical approval The article is an editorial and does not involve any human or animal studies requiring ethical approval.

Informed consent For this type of article informed consent is not required.

\section{References}

1. Bligh, J. (2002). The first year of doctoring: still a survival exercise. Medical Education, 36(1), 2-3.

2. Safir, O., Sonnadara, R., Mironova, P., \& Rambani, R. (Eds.). (2018). Boot camp approach to surgical training (1st ed.). Amsterdam: Elsevier Inc.

3. Rambani, R., Viant, W., Ward, J., \& Mohsen, A. (2013). Computer-assisted orthopedic training system for fracture fixation. Journal of Surgical Education, 70(3), 304-308.

4. Cleland, J., Walker, K. G., Gale, M., \& Nicol, L. G. (2016). Simulation-based education: understanding the socio-cultural complexity of a surgical training 'boot camp'. Medical Education, 50(8), 829-841.

5. Cleary, D. R., Siler, D. A., Whitney, N., \& Selden, N. R. (2018). A microcontroller-based simulation of dural venous sinus injury for neurosurgical training. Journal of Neurosurgery, 128(5), $1553-1559$.

Publisher's Note Springer Nature remains neutral with regard to jurisdictional claims in published maps and institutional affiliations. 